\title{
$250 \mathrm{kA}$ compact linear transformer driver for wire array $z$-pinch loads
}

\author{
S. C. Bott, D. M. Haas, R. E. Madden, U. Ueda, Y. Eshaq, G. Collins IV, K. Gunasekera, D. Mariscal, \\ J. Peebles, and F. N. Beg \\ University of California San Diego, La Jolla, California 92093, USA
}

M. Mazarakis, K. Struve, and R. Sharpe

Sandia National Laboratories, Albuquerque, New Mexico 87185-1194, USA

(Received 22 March 2011; published 27 May 2011)

\begin{abstract}
We present the application of a short rise ( $\sim 150 \mathrm{~ns}) 250 \mathrm{kA}$ linear transformer driver (LTD) to wire array $z$-pinch loads for the first time. The generator is a modification of a previous driver in which a new conical power feed provides a low inductance coupling to wire loads. Performance of the new design using both short circuit and plasma loads is presented and discussed. The final design delivers $\sim 200 \mathrm{kA}$ to a wire array load which is in good agreement with SCREAMER calculations using a simplified representative circuit. Example results demonstrate successful experiments using cylindrical, conical, and inverse wire arrays as well as previously published work on $x$-pinch loads.
\end{abstract}

DOI: 10.1103/PhysRevSTAB.14.050401

\section{INTRODUCTION}

The development of linear transformer driver (LTD) technology [1-3] for generating short, high current pulses represents a significant advance in the pursuit of the $z$-pinch-driven approach to inertial fusion energy (IFE) [4-7]. This design eliminates the need for large volumes of insulating oil and water which is required for present Marx bank driven generators, and a single compact unit can deliver significant current ( $>1 \mathrm{MA})$. This is achieved by the parallel discharge of capacitors arranged around a central electrode, with the capacitance and internal inductances of the capacitors themselves determining the output rise time, rather than the use of water-insulated pulse-forming lines. Each capacitor may be of order $10-100 \mathrm{nF}$, facilitating a short, $\sim 100 \mathrm{~ns}$, quarter period of the discharge cycle ("rise time"). Capacitors are typically arranged in pairs with a connecting switch, referred to as "bricks," and a unit (termed a "cavity") comprises a number of bricks. Voltages in a single cavity are low $(\leq 100 \mathrm{kV})$, but high output voltage can be achieved inductively adding several units in series. Large systems are therefore entirely modular, and can be constructed of the required number of parallel and series units to achieve the desired current and voltage characteristics. In effect for a similar floor area, the LTD design increases the stored energy density by a factor of $\sim 2$, and the output power cost (/Watt) can be significantly reduced compared to present Marx-based systems.

Published by the American Physical Society under the terms of the Creative Commons Attribution 3.0 License. Further distribution of this work must maintain attribution to the author(s) and the published article's title, journal citation, and DOI.
PACS numbers: 29.20.- c, 52.58.Lq, 84.30.Ng, 84.60.Ve

This modular design is particularly attractive for several reasons: first, it allows design and testing of individual bricks, and then cavities before assembly into large generators, which is not possible for Marx driven systems. Second, the large number of small components in a very large generator permits a "soft-fail" mechanism. With several hundred thousand capacitors and switches, and a few hundred cavities, the failure of an individual capacitor, switch, brick, or even cavity does not significantly affect the overall generator performance. Defective units can be simply removed and replaced with reserve components. In addition, each cavity, brick, or any combination of both can be triggered independently, allowing for an extremely flexible output current waveform. Such close control is required for several types of investigation, including the behavior of materials under isentropic compression [8-10] which has been extremely successful recently.

The LTD design also has one crucial advantage over high energy storage Marx bank, pulse-forming line systems; it can be repetition rated. This is not achievable for present designs, such as the $Z$ machine at Sandia National Laboratories, and is the single biggest advance in recent times for current rise times of $\sim 100 \mathrm{~ns}$. Recent studies by Mazarakis et al. [11,12] have demonstrated the operation of $0.5 \mathrm{MA}$ cavities at repetition rates of $\sim 0.1 \mathrm{~Hz}$, which is the rate required for $z$-pinch driven IFE power station designs [4-6]. A detailed discussion comparing very high power LTD and Marx systems is given in [13]

In this paper, we present the completion of a $250 \mathrm{kA}$ LTD generator at the University of California San Diego. The device has been named GenASIS (Generator for Ablation Structure and Implosion Studies), and represents the first application of a short rise time LTD unit for the analysis of wire array $z$-pinch systems. The main content of this paper is as follows: Sec. II describes the generator 
configuration and operation, Sec. III describes the modification of the power feed to provide a low inductance coupling to wire loads, Sec. IV presents and discusses load current scaling with charge voltage for short circuit loads, Sec. V presents demonstration of wire array $z$-pinch loads, and Sec. VI provides a summary.

\section{LTD CAVITY CONFIGURATION AND OPERATION}

The LTD cavity is a square design developed by the Institute for High Current Electronics in Tomsk, Russia [14], and the basic layout is given in Fig. 1. The cavity uses $12 \times 20 \mathrm{nF}$ double ended general atomics capacitors (model No. 35467), which are grouped around the central discharge plate in blocks of three, each of which is connected to a switch block. The switches themselves comprise multiple rows of spherical brass electrodes, which are capacitively triggered using a small Marx unit built into the main body of the cavity. The gas insulation in the switches is atmospheric air, and no air flow is required during normal operation. Potentially, each switch block can be triggered independently, but at present all four are triggered by a single pulse from the trigger Marx. The Marx output is $\sim 70 \mathrm{kV}$ with a rise time of $\sim 25 \mathrm{~ns}$ and is connected to one row of each of the switch blocks through an insulated conductor cord. The Marx generator is itself triggered by a $20 \mathrm{kV}$ thyratron unit which is mounted external to the cavity.

During operation, the thyratron, the trigger Marx unit, and the main cavity capacitors are all charged through a single $+100 \mathrm{kV}$ dc Glassman power supply, which is isolated from voltage reversal through a high voltage diode chain (Glassman ADC-SNL series, $200 \mathrm{kV}$-rated) immersed in transformer oil. The low capacitance allows a full charge to $80 \mathrm{kV}$ in approximately 13 seconds. When the machine is fully charged a master switch is closed, and this triggers a series of delay boxes which fire both the cavity and the diagnostics. One trigger is sent to the thyratron unit, which fires the trigger Marx, and subsequently the main switches, and the main capacitors then discharge through the load. The switches allow the positive high voltage to connect to ground, sending a negative voltage waveform to the central discharge plate, which is therefore the cathode. The whole sequence from thyratron output to peak current at the load takes $\sim 700 \mathrm{~ns}$ (see Fig. 1).

The UCSD generator has a rather higher impedance than some cavities, at $\sim 0.3 \Omega$, meaning it is more suitable for driving $z$-pinch and $x$-pinch loads which involve rapid

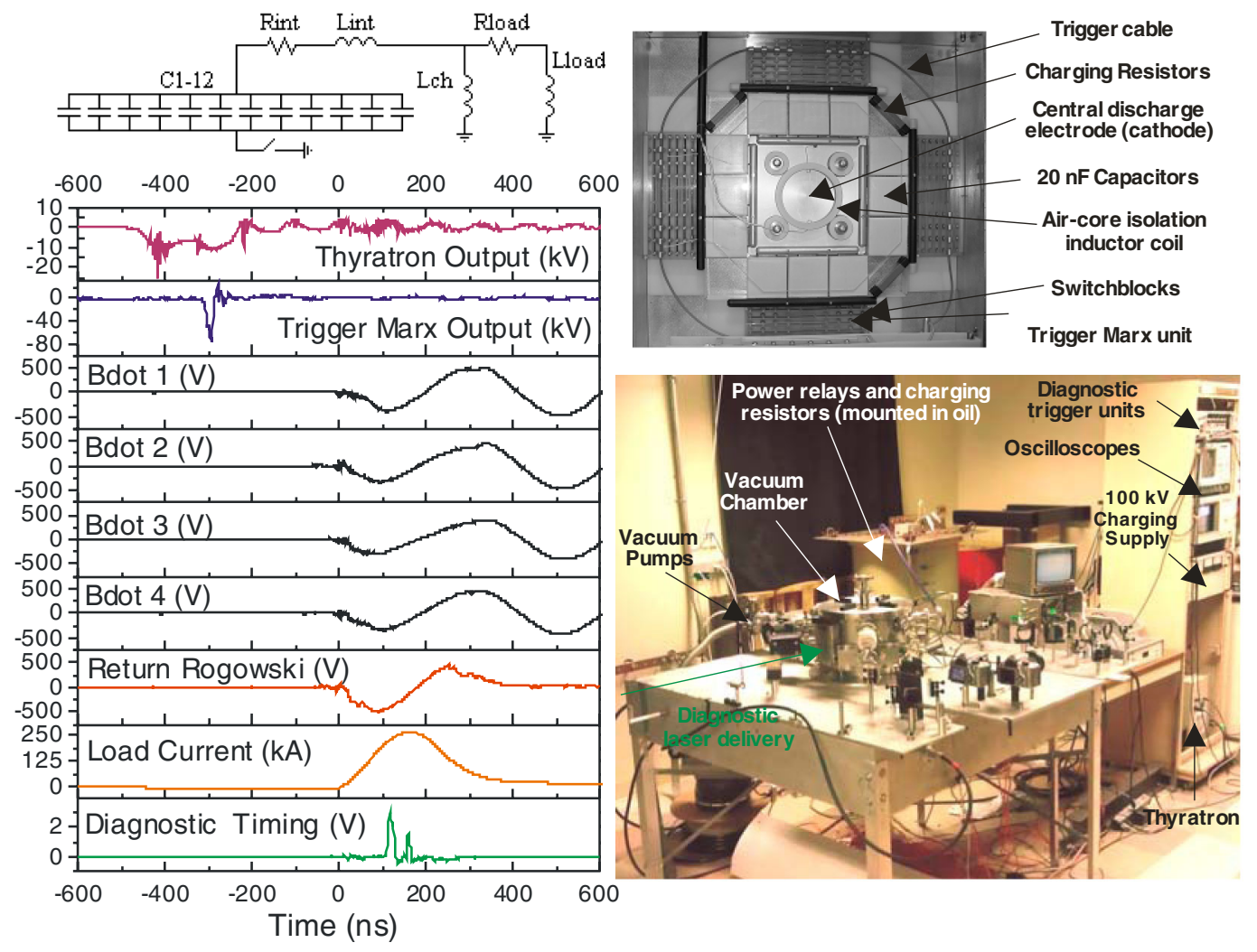

FIG. 1. Present configuration of the GenASIS LTD driver at UC San Diego. The circuit diagram is the representative model used for circuit simulations, plots show timings for electrical diagnostics during the trigger sequence, the upper image shows the interior of the cavity, and the lower image shows the device in the laboratory. 
changes of inductance. Additionally, the isolation inductor is air cored, rather than iron cored as is used for the higher current version of the device, although this still provides adequate isolation during the drive time. The cavity measures only $1.24 \times 1.24 \times 0.23 \mathrm{~m}^{3}$, and the charging, triggering, and data acquisition equipment is mounted in a 19 " rack mount, with the high voltage (HV) diode isolation unit a similar size. Since the outside of the cavity is always at ground and both the trigger Marx and the main switches and capacitors are completely enclosed, this can be located relatively close to the machine. This compact size, along with the lack of pressurized gas switches, large volumes of oil in the cavity itself, and no mega-volt potentials, make such a device ideal for a small university laboratory.

\section{CONICAL POWER FEED AND EXPERIMENTAL CHAMBER DESIGN}

An experimental vacuum chamber was designed and constructed at UC San Diego to allow diagnostics access to plasma loads. The stainless steel chamber is $10^{\prime \prime}$ in diameter and 6 " high which provides two levels of diagnostic access though NW40 vacuum ports on two levels. Four of the NW40 ports were substituted for square ports in horizontal and vertical orientations, to give both "wide" and "tall" laser access to experiments. The vacuum chamber uses a single $70 \mathrm{l} / \mathrm{s}$ turbomolecular pump (Edwards EXT70), and typically achieves operating pressures of $\sim 10^{-5}$ mbar in approximately 20 minutes.

The development of the electrode configuration served two purposes. First, it was anticipated from analytical modeling and practical observation of the plasma, that load diameters of $10 \mathrm{~mm}$ would be suitable to study wire ablation phenomena in various configurations. To install such loads and maintain a high current level, the electrode diameters needed to be reduced from the initial $\sim 150 \mathrm{~mm}$ to approximately $20 \mathrm{~mm}$ (to allow for mounting of load hardware) at a low inductance "cost." Second, the load needed to be raised to allow diagnostic access with the new vacuum chamber attached. In order to achieve this, a coaxial conical power feed with constant electrode gap was designed and constructed in-house. The use of a constant gap in the cone is not the most efficient design (as discussed below) but allowed ease of machining and positioning as an initial design. Discussions with Gomez et al. [15,16] aided in the design of the conical section.

The configuration comprises three parts: a coaxial cylinder to raise the load, and coaxial conical to reduce the diameter, and finally a cylindrical wire array load. The wire load was modeled as a uniform cylinder with four discrete return posts (as in the experiment) to allow optimization of the current return diameter. The total inductance of these elements is

$$
L_{\text {total }}=L_{\text {cyl }}+L_{\text {cone }}+L_{\text {array }}
$$

$$
\begin{aligned}
L_{\text {total }}= & 2 l_{\text {cyl }} \ln \left(\frac{r_{\text {outer,cyl }}}{r_{\text {inner,cyl }}}\right)+2\left(\frac{\Delta}{\cos \theta}\right) \ln \left(\frac{r_{\text {initial,cone }}}{r_{\text {final, cone }}}\right) \\
& +\left(2 l_{\text {array }}\left[\ln \left(\frac{r_{\text {return }}}{r_{\text {array }}}\right)+\frac{1}{N}\left(\frac{r_{\text {return }}}{N r_{\text {posts }}}\right)+\operatorname{lns}+\frac{1}{4 N}\right]\right),
\end{aligned}
$$

where the following dimensions were applied: $L_{\text {cyl }}=$ length of cylindrical section $=80 \mathrm{~mm}, R_{\text {outer,cyl }}=$ outer radius of cylinder $=75 \mathrm{~mm}, R_{\text {inner,cyl }}=$ inner radius of cylinder $=70 \mathrm{~mm}, \Delta=$ electrode gap in cone section $=$ $4 \mathrm{~mm}, \quad \theta=$ cone angle $=40^{\circ}, \quad r_{\text {initial, cone }}=$ cone radius at base $=75 \mathrm{~mm}, \quad r_{\text {final, cone }}=$ cone final radius at load $=$ $10 \mathrm{~mm}, l_{\text {array }}=$ array length $=10 \mathrm{~mm}, r_{\text {return }}=$ return post diameter $=25 \mathrm{~mm}, r_{\text {array }}=$ array diameter $=5 \mathrm{~mm}, N=$ number of return posts $=4, \quad r_{\text {posts }}=$ radius of each return post $=2 \mathrm{~mm}, \zeta=$ geometry factor [17-20]. Using these values, $L_{\text {total }}=7.1 \mathrm{nH}$. This value is relatively insensitive to cone angle, cylindrical section (inner and outer radii being fixed by the initial electrode spacing on the LTD unit), with critical values being the array return current radius (assuming a $10 \mathrm{~mm}$ load diameter and height), and the electrode gap in the cone. SCREAMER [21] circuit modeling suggested the above design parameters should deliver $\sim 200 \mathrm{kA}$ to wire loads. Modeling used a simple equivalent circuit, without considering core losses as in Ref. [11]. The final power feed design is given in Fig. 2.

The electrode gap was incremented over a series of short circuit shots to check for surface flashover at high charging voltages. This was eventually set at $\sim 4 \mathrm{~mm}$ and shows no arcing or discoloration on inspection after several loads at $>70 \mathrm{kV}$. The field strength across the electrode gap in the feed is therefore a maximum of $200 \mathrm{kV} / \mathrm{cm}$ (at $V_{\mathrm{ch}}=$ $80 \mathrm{kV}$ ). This is approximately the electric field limit for well finished electrodes, but the self-generated B-field of the power feed during the current pulse, particularly close to the load, provides some magnetic insulation. Using the limit to onset of magnetic insulation described in [22], the load end of the cone section is insulated from early times given the small radius here. Only a few kA

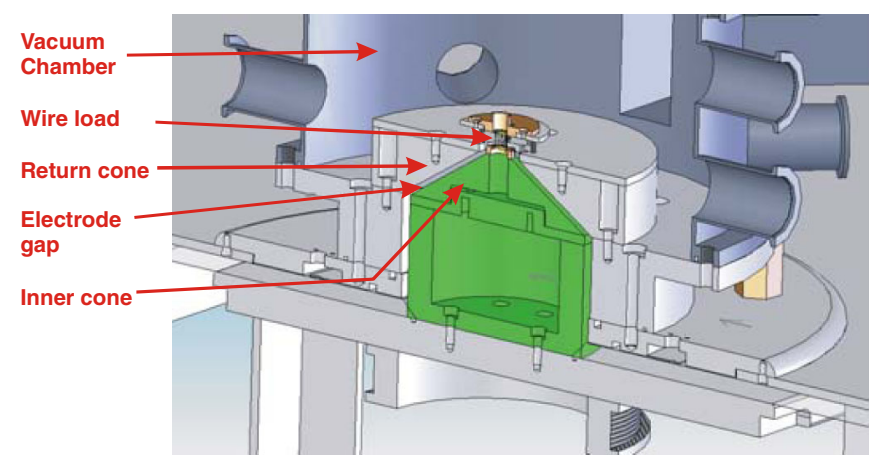

FIG. 2. CAD drawings of the completed conical power feed (inner cone in green and return structure in grey), showing wire array load. 
(i.e. very early in the rise time) is sufficient to prevent electron loss across the gap. At the base of the cone, however, the larger radius means that magnetic insulation occurs at around half the rise time of the current, assuming a worst case scenario of the full charge voltage $80 \mathrm{kV}$ across a $4 \mathrm{~mm}$ gap from $t=0$. A tapered power feed gap, beginning at $\sim 10 \mathrm{~mm}$ and finishing at the load at $\sim 2 \mathrm{~mm}$, would provide the ideal geometry to ensure magnetic insulation throughout the feed from early time. This may be attempted in future iterations of the generator design. The comparison of measured load and machine currents carried out in Sec. V suggests the current fixed gap cone operates sufficiently well at voltages up to $75 \mathrm{kV}$.

\section{SHORT CIRCUIT TESTING}

The cavity was originally designed to be operated at $60-80 \mathrm{kV}$, and a series of shots were carried out to determine the performance of the cavity as a function of charge voltage with the new power feed attached. Since maximizing the load current in a regime of reliable operation is the principle aim here, tests were carried out between 68 and $80 \mathrm{kV}$. As noted in Ref. [15], the electrode gaps in the trigger Marx unit must be adjusted manually to facilitate voltage hold-off until trigger, and incorrect adjustment is one of the main causes of prefire in this device. It should also be noted that, since the Marx unit is also insulated only by atmospheric air, the exact adjustment will be sensitivity to the local altitude and humidity, and this was determined empirically.

The short circuit load is a $\frac{1}{2}$ steel bolt which connects the anode plate directly to the cathode using a threaded cathode piece. Current is measured in two positions relative to the load. The first is a groove machined into the return plate of the cavity itself. This serves to measure current at any position across the insulator between the cathode and return structure. There are four outputs from this groove which are located centrally to each of the four sides of the square cavity. These outputs are termed
"Machine Bdots" (MBdot1-4). These are nominally identical, and typically agree to within a few percentage points. In the case of a flashover somewhere in the power feed, these outputs will report the full current delivered, but not that this current necessarily reached the load at the end of the new power feed design. Therefore two multiturn Rogowski coils were fabricated and each mounted on one of four return posts (referred to as "return Rogowskis" 1 and 2). The signals from these, accounting for attenuation and sensitivity, can be directly compared to the machine Bdot signals to determine if the load sees the full current or shorting is occurring. This comparison allows us to determine if the power feed is operating as desired.

Figure 3 shows the variation of the load current and the $1 \sigma$ standard deviation as a function of the machine charge voltage. At each charge voltage the Marx unit gap was optimized, and each point on the plot represents ten shots. The load current is taken here as the average of the four machine Bdots, provided the return Rogowskis show no short across the power feed has occurred. The load current varies linearly with charge voltage, from $\sim 195 \mathrm{kA}$ at $68 \mathrm{kV}$ to $\sim 260 \mathrm{kA}$ at $80 \mathrm{kV}$. The standard deviation generally decreases rapidly for charge voltages $>70 \mathrm{kV}$ becoming $\sim 2 \%$ for $V_{\text {ch }}=80 \mathrm{kV}$, likely a result of the high voltage providing a more reliable breakdown at the main switch blocks. Noticeably, both $V_{\text {ch }}=72 \mathrm{kV}$ and $75 \mathrm{kV}$ show surprisingly small deviations from the mean current, at $1.4 \%$ and $1.2 \%$, respectively. It is not clear presently why this should be the case. The efficiency of the generator can be estimated by comparing the inductive energy of the current drive $\left(\frac{1}{2} L_{\text {total }} I_{\text {load }}^{2}\right)$ with the initial stored energy in the capacitors $\left(\frac{1}{2} C_{\text {total }} V_{\mathrm{ch}}^{2}=768 \mathrm{~J}\right.$ for $\left.V_{\mathrm{ch}}=80 \mathrm{kV}\right)$. This is $\sim 30 \%$ for all charge voltages. It should be noted that the efficiency here is limited by the relatively high impedance of this generator, and recent work by Kim et al. [23] has demonstrated efficiencies of $\sim 70 \%$ for 1 MA LTD cavities.

The most likely components in the cavity to affect the repeatability of the current drive are the switch blocks. To
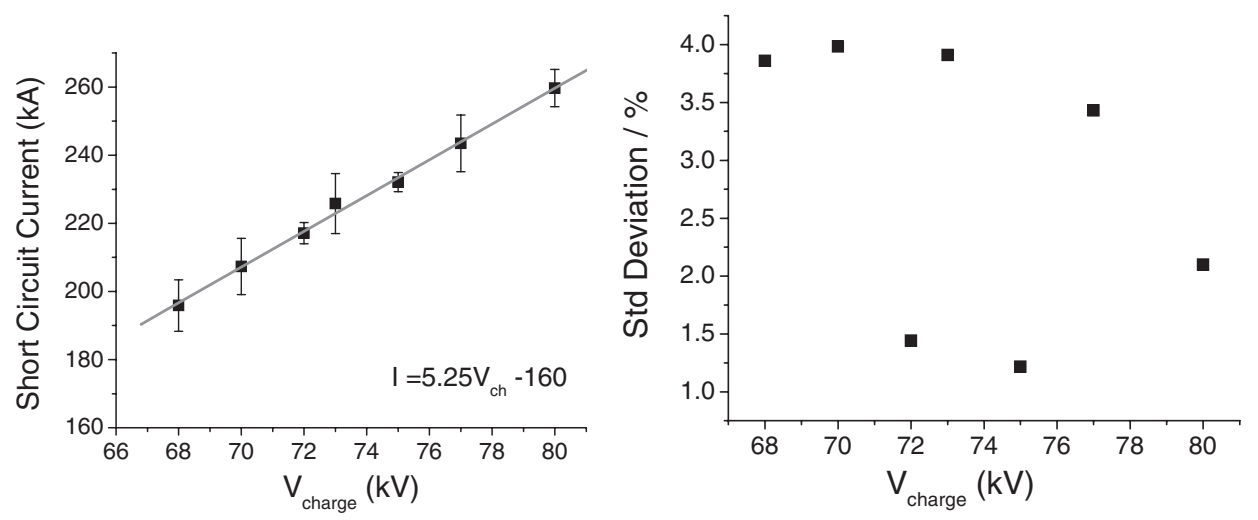

FIG. 3. Plot for short circuit loads showing (left) variation of mean load current and (right) variation of standard deviation as a function of machine charge voltage. 

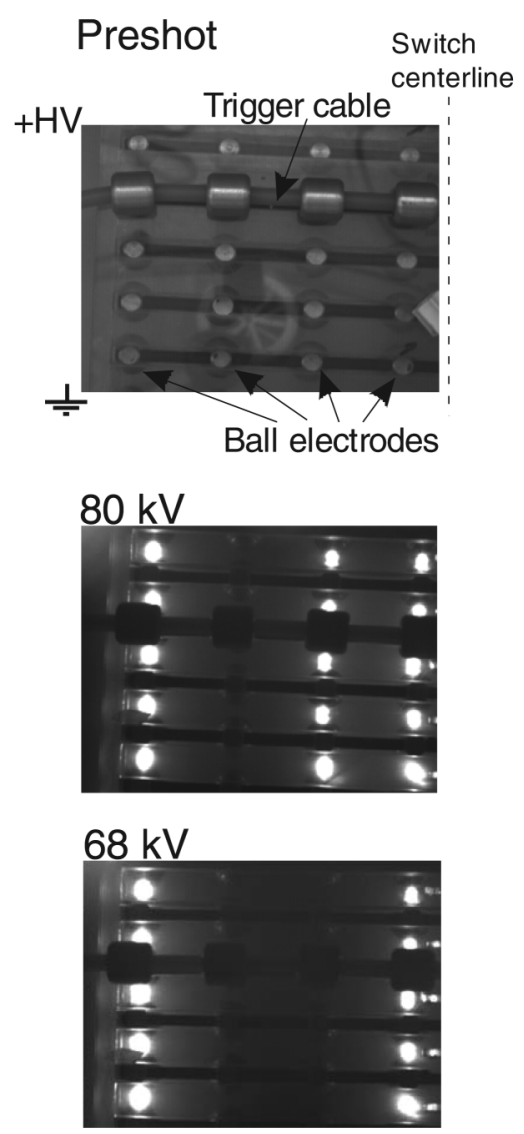

FIG. 4. Preshot and time-integrated images of switch block operation at different charge voltages (emission to the right of discharges on rightmost row is reflection from the Perspex cover on the switch block).

provide a preliminary investigate of the switch operation, a CCD camera was mounted to image one half of a block (i.e., four of the eight rows connecting HV to ground). The shutter was open for the entire experiment, and so provides time-integrated information about which switches fired in a particular shot. Example images are given in Fig. 4.

These images can be used to assess the number of channels which operate for a given shot, and hence the inductance of the switch section as a whole. For ten shots at each charge voltage, the number of channels observed to fire was recorded, and these data are given in Fig. 5. The number of channels of the switches which break for a particular shot increases slightly with increasing charge voltage as would be expected. It is interesting to note that even at the maximum design charge of $80 \mathrm{kV}$ not all channels are used, indicating that the lowest switch inductance possible is not attained. To estimate the effect of the channel number on the operation of the cavity, we can estimate the difference in inductance introduced by the variable switch operation. The inductance of a multichannel switch in nano-Henries is given by [24]

$$
L_{\text {switch }}=\frac{2 L}{N} \ln \left(\frac{D}{s}\right)
$$

where $L, D$, and $s$ are the length, distance between channels divided by 3 , and channel diameter, respectively, in $\mathrm{cm}$, and $N$ is the number of channels which are observed to operate. To estimate the effect of changing $N$ we must make some assumptions about the measurements we can obtain from the types of images given in Fig. 4. We image one half of a total of four switch blocks. We therefore assume that each of the other seven halves operate identically to the one we image, and so simply multiply the number of channels we observe to be used by 2 to obtain $N$ for one switch block, and assume the other three switches block use the same value for $N$.

From the imaging data, we can set $L=6 \mathrm{~cm}, D=$ $4.5 \mathrm{~cm} / 3=1.5 \mathrm{~cm}$, and $s=0.75 \mathrm{~cm}$. Since all the switch blocks are mounted in parallel, we can treat them as a single unit with a total of 32 rows of electrodes. We can
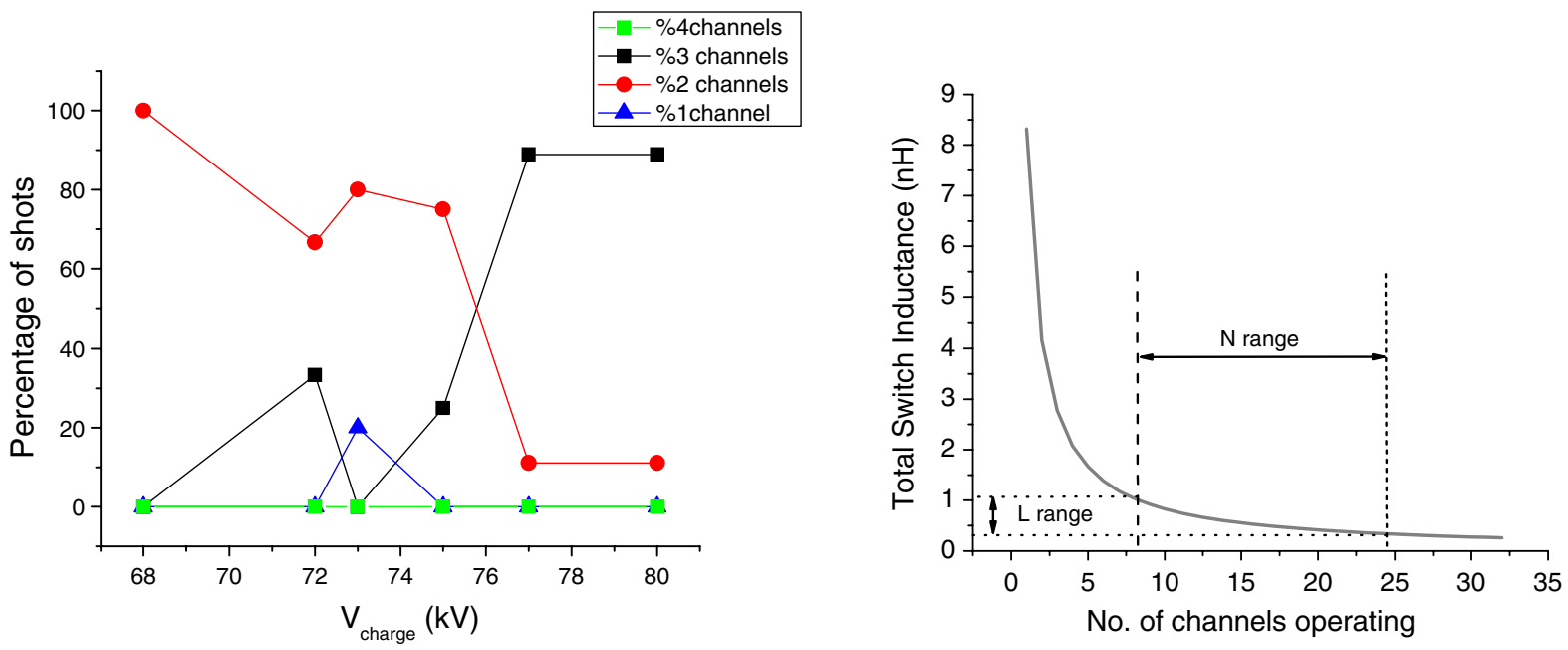

FIG. 5. Inductance as a function of channel number $N$, and variation of number of channels observed to operate as a function of charge voltage. 

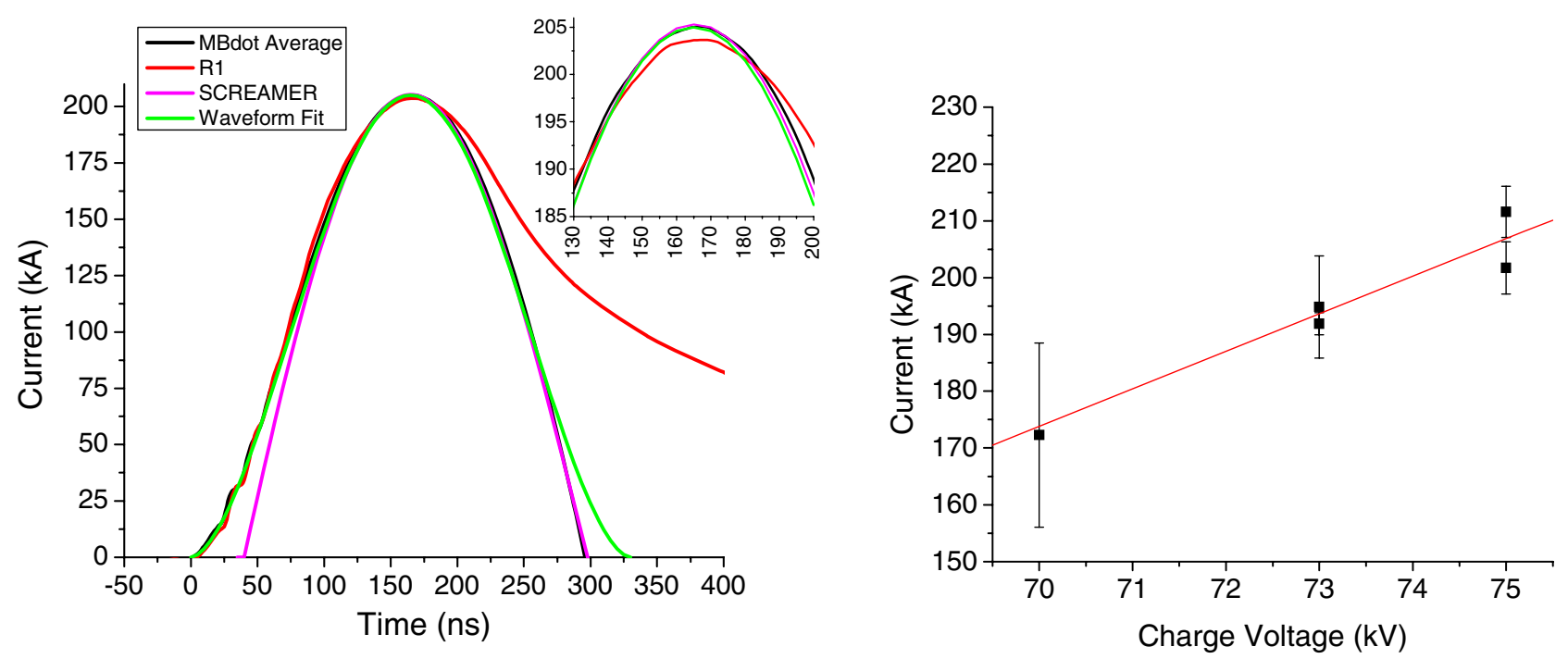

FIG. 6. (left) Comparison of machine Bdot current and return path Rogowski coil currents to SCREAMER output and a waveform fit [Eq. (4)] for a cylindrical wire array load, and (right) scaling of load current with charge voltage for cylindrical array.

then calculate the average (i.e., time independent) inductance of the switches, and this plot is given in Fig. 5. The plots show that there is some variation in the number of channels observed to operate. Typically at $68 \mathrm{kV}$ only two of the four observed channels fire, implying that only 16 of the total of 32 possible channels fire. As charge voltage increases, eventually $\sim 90 \%$ of shots use three channels (24 of 32). It is interesting to note that no images throughout the test period showed that all channel were in operation during a shot. Despite the variation in $N$, the other plot shows that the total inductance of the switch section does not change by very much. At eight out of 32 channels, the inductance is $1.04 \mathrm{nH}$ and at 24 out of 32 this is reduced only to $0.35 \mathrm{nH}$. Whilst this is a reduction of a factor of 3 , given the inductance of the remainder of the system (capacitor inductance power feed, etc.) is of order $20 \mathrm{nH}$, this change is likely to be insignificant. Therefore, provided that at least one channel on each switch block fires, the maximum current is unaffected by variation in the total $N$ for a particular shot.

A much fuller investigation would be needed to address at which point in the current drive each channel closes and whether closure time scale is an issue. This may allow an analysis of the time varying inductance and resistance of the switch during the main current drive time. Presently we are mounting fiber optics to each row of switches in the machine to determine more accurately how many fire and at what time each row closes. These studies will be reported in a future publication.

\section{WIRE ARRAY LOAD RESULTS}

The testing of cylindrical wire arrays was carried out to determine the load current maximum as a function of charge voltage, and assess if the design of the power feed provides the desired current level $(>200 \mathrm{kA})$. For this section, loads comprised eight wires on a $10 \mathrm{~mm}$ diameter of either $10 \mu \mathrm{m} \mathrm{W}$ or $25 \mu \mathrm{m} \mathrm{Al}$. The load current was measured by the "return" Rogowski coils mounted around two of four return current posts. These are compared to the averaged machine Bdot probes to determine the load current and effective insulation of the power feed. A typical comparison at a charge voltage of $75 \mathrm{kV}$ is shown in Fig. 6. The return Rogowski coils typically agree with the machine Bdot current to $<3 \%$ until shortly after the time of peak current, and the SCREAMER output agrees very well with the data for a $7 \mathrm{nH}, 0.01 \Omega$ load. The variation of load current with charge voltage has also been investigated, and is summarized in Fig. 6. The load current is well described by Eq. (4), which allows estimation of the ablated plasma profiles from analytical modeling:

$$
I(t)=I_{\max }\left[\sin \left(\frac{\pi t}{2 \tau}\right)^{1.7}\right] .
$$

Since completion, GenASIS has been used to drive a variety of wire loads, including cylindrical arrays, conical arrays [25] and $x$-pinches [26], planar and inverse wire arrays with currents $\sim 200 \mathrm{kA}$. Figure 7 shows some example results from recent experiments. Figure 7(a) shows the ablation phase of a tungsten wire array conical using gated soft x-ray self-emission. The development of the ablation flare structure, common to all exploding wire experiments where a global B-field is present, can be clearly observed. Figure 7(b) shows dark field laser schlieren images of a double planar wire array in which early plasma formation is streaming towards the global axis, eventually forming an MHD unstable plasma column. Figure 7(c) shows laser interferometry images of a tungsten inverse wire array, where the flare structure can be 


\section{a) Conical Wire Array $(8 \times 19 \mu \mathrm{m} \mathrm{W})$}

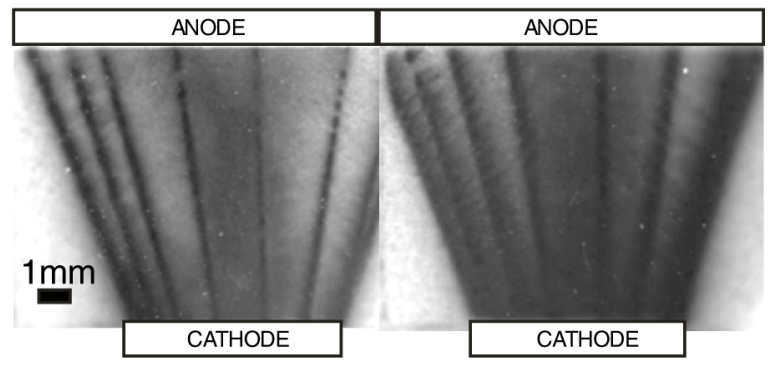

b) Double Planar Wire Array $(2 \times 4 \times 25 \mu \mathrm{m} \mathrm{Al})$

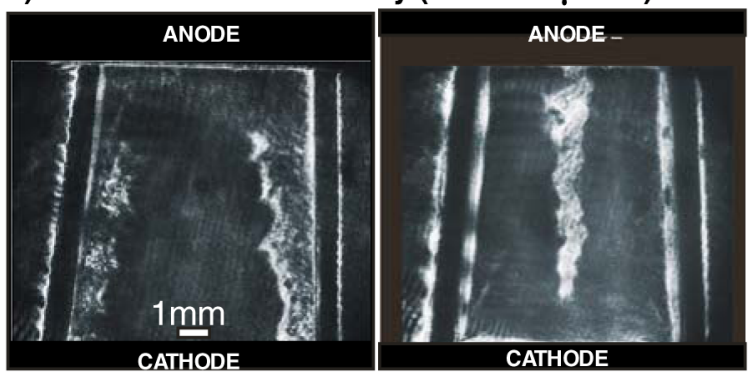

c) Inverse Wire Array ( $2 \times 10 \mu \mathrm{m} \mathrm{W})$

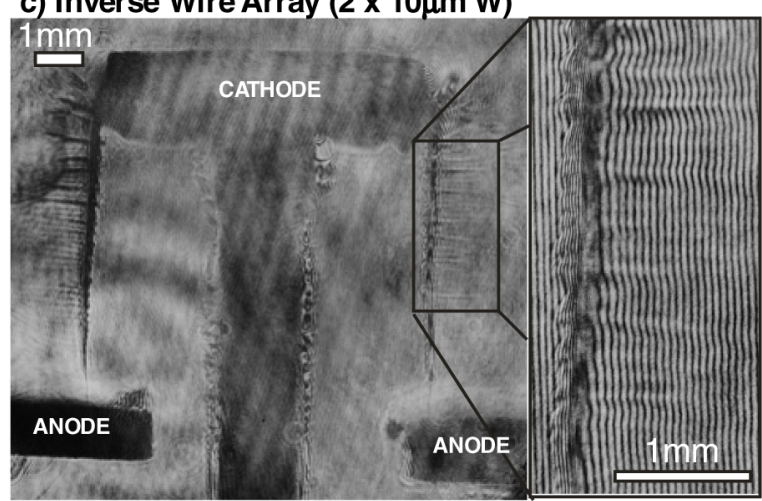

FIG. 7. Examples of results on the $200 \mathrm{kA}$ GenASIS machine: (a) conical wire array, (b) double planar wire array, and (c) inverse wire array.

observed with sufficient detail to enable future quantitative analysis.

Over a period of 2 months during the short circuit and wire load testing, the cavity was fired 179 times (including 60 over 2 days) with only a few prefires, and almost all of these used $80 \mathrm{kV}$ charge voltage. For experiments, the cavity is typically operated at $75 \mathrm{kV}$ with Marx gap optimized for this, and failure rate (including flashover at electrodes and prefires) is very low, of order a few percentage points. The machine can fire many times per day, up to 10 for simple experiments, and requires very little maintenance. Turn on time to fire is limited by diagnostic checks and thyratron warm-up. The power feed is easily removed for inspection, and is typically cleaned every few weeks, mainly as a result of debris buildup at the load end since this is open directly below the exploding wire.

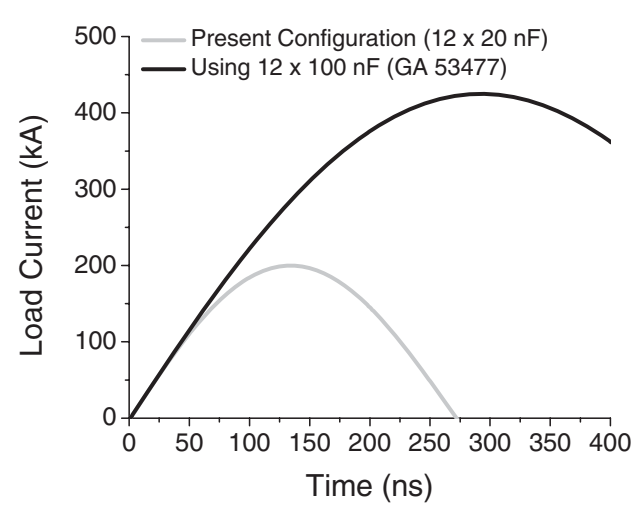

FIG. 8. SCREAMER outputs comparing the present configuration of GenASIS with a larger capacitance configuration.

One interesting modification that may be achieved is the use of different capacitors in the cavity to optimize of the current drive for different experiments. For example, the present capacitors could be replaced with the GA model $35477100 \mathrm{nF}$ capacitors. The additional capacitance at a similar inductance could allow peak currents of $>400 \mathrm{kA}$ with a rise time of $\sim 300 \mathrm{~ns}$ (Fig. 8). In terms of the mass ablated from a wire array, which is proportional to the integral of the square of the drive current $\left(\int I^{2}\right)$ and "useful" up to peak current, a factor of 20 would be gained. Of course, modifications would be needed to house these larger and heavier capacitors in the cavity as well as construct a suitable electrical contact, but there is sufficient room below the machine in its current configuration. The simplicity of the cavity and low voltage operation make such things feasible.

\section{SUMMARY}

The GenASIS linear transformer driver has been completed by the development and testing of a new conical power feed. The generator delivers $200 \mathrm{kA}$ to wire loads in $150 \mathrm{~ns}$, in line with circuit modeling. Successful experiments have been carried out for a range of wire array $z$-pinch loads. In the future we will explore the possibility of repetition rating the device to study the behavior of power feeds under this regime, and to investigate means of power feed reload systems to support the realization of $z$-pinch driven IFE.

\section{ACKNOWLEDGMENTS}

The UC San Diego team is grateful to Sandia National Laboratories and to Dr. Chris Deeney for both the loan of the generator and the technical support in completing its installation. The UCSD authors also thank Mr. Sean McGraw (Ashland University, Ohio) who helped with some of the laboratory testing during his National Undergraduate Fellowship (NUF) placement. This work was supported by the Department of Energy under Contract No. DE-SC-0001063. 
[1] M. G. Mazarakis and R. B. Spielman, in Proceedings of the 12th IEEE International Pulsed Power Conference, edited by C. Stallings and H. Kirbie (IEEE, Piscataway, NJ, 1999), p. 412.

[2] A. A. Kim, A. N. Bastrikov, S. N. Volkov, V. G. Durakov, B. M. Kovalchuk, and V. A. Sinebryukhov, in Proceedings of the 13th International Symposium on High Current Electronics, edited by Boris Kovalchuk and Gennady Remnev (IHCE SB RAS, Tomsk, Russia, 2004), p. 141.

[3] M. G. Mazarakis, W.E. Fowler, F.W. Long, D. H. McDaniel, C. L. Olson, S. T. Rogowski, R. A. Sharpe, and K.W. Struve, in Proceedings of the 15th IEEE International Pulsed Power Conference, edited by J.E. Maenchen and E. Schamiloglu (IEEE, Piscataway, NJ, 2005), p. 390.

[4] M. G. Mazarakis and C. L. Olson, Proceedings of the 21st IEEE/NPSS Symposium on Fusion Engineering (SOFE 2005) (IEEE, Piscataway, NJ, 2005), pp. 02-09.

[5] C.L. Olson et al., Sandia National Laboratory Report No. SAND-2005-2742P, 2005.

[6] C. L. Olson et al., Sandia National Laboratory Report No. SAND-2006-7399P, 2006.

[7] M. Keith Matzen, M. A. Sweeney, R. G. Adams, J. R. Asay, J.E. Bailey, G. R. Bennett, D.E. Bliss, D. D. Bloomquist, T.A. Brunner, R. B. Campbell, G. A. Chandler, C. A. Coverdale, M.E. Cuneo, J.-P. Davis, C. Deeney, M. P. Desjarlais, G. L. Donovan, C. J. Garasi, T. A. Haill, C. A. Hall, D. L. Hanson, M. J. Hurst, B. Jones, M. D. Knudson, R. J. Leeper, R. W. Lemke, M. G. Mazarakis, D. H. McDaniel, T. A. Mehlhorn, T. J. Nash, C. L. Olson, J. L. Porter, P. K. Rambo, S. E. Rosenthal, G. A. Rochau, L. E. Ruggles, C. L. Ruiz, T. W. L. Sanford, J. F. Seamen, D. B. Sinars, S. A. Slutz, I. C. Smith, K. W. Struve, W. A. Stygar, R. A. Vesey, E. A. Weinbrecht, D. F. Wenger, and E.P. Yu, Phys. Plasmas 12, 055503 (2005).

[8] D.E. Bliss et al., in Proceedings of the 14th IEEE International Pulsed Power Conference, edited by $\mathrm{M}$. Giesselmann and A. Neuber (IEEE, Piscataway, NJ, 2003), p. 179.

[9] R. W. Lemke, M. D. Knudson, A. C. Robinson, T. A. Haill, K. W. Struve, T. A. Mehlhorn, and J.R. Asay, in Proceedings of the Fifth International Conference on Dense Z-Pinches, edited by J. Davis, C. Deeney, and N. R. Pereira (AIP, Melville, NY, 2002), p. 299.

[10] C. W. Greeff, P. A. Rigg, M. D. Knudson, R. S. Hixson, G. T. Gray, III, in Shock Compression of Condensed Matter-2003, edited by M.D. Furnish, Y.M. Gupta, and J. W. Forbes (AIP, New York, 2003), p. 209.

[11] M. G. Mazarakis, W.E. Fowler, K. L. LeChien, F. W. Long, M. K. Matzen, D. H. McDaniel, R. G. McKee, C. L. Olson, J. L. Porter, S. T. Rogowski, K. W. Struve, W. A. Stygar, J.R. Woodworth, A. A. Kim, V. A. Sinebryukhov, R. M. Gilgenbach, M. R. Gomez, D. M.
French, Y. Y. Lau, J.C. Zier, D. M. VanDevalde, R. A. Sharpe, and K. Ward, IEEE Trans. Plasma Sci. 38, 704 (2010).

[12] Michael G. Mazarakis, William E. Fowler, Alexander A. Kim, Vadim A. Sinebryukhov, T. Rogowski, Robin A. Sharpe, Dillon H. McDaniel, Craig L. Olson, John L. Porter, Kenneth W. Struve, William A. Stygar, and Joseph R. Woodworth, Phys. Rev. ST Accel. Beams 12, 050401 (2009).

[13] W. A. Stygar, M. E. Cuneo, D. I. Headley, H. C. Ives, R. J. Leeper, M. G. Mazarakis, C. L. Olson, J. L. Porter, T. C. Wagoner, and J.R. Woodworth, Phys. Rev. ST Accel. Beams 10, 030401 (2007).

[14] V. Kharlov, B. M. Kovalchuk, and V. B. Zorin, Rev. Sci. Instrum. 77, 123501 (2006).

[15] R. M. Gilgenbach, M. R. Gomez, J. C. Zier, W. W. Tang, D. M. French, Y. Y. Lau, M. G. Mazarakis, M.E. Cuneo, M. D. Johnston, B. V. Oliver, T. A. Mehlhorn, A. A. Kim, and V.A. Sinebryukhov, AIP Conf. Proc. 1088, 259 (2009).

[16] M. R. Gomez, R. M. Gilgenbach, Y. Y. Lau, W. Tang, J. C. Zier, M. G. Mazarakis, M. E. Cuneo, T. A. Mehlhorn, and W. A. Stygar, in the 16th IEEE International Pulsed Power Conference, Albuquerque, NM (IEEE, Piscataway, NJ, 2007), p. 152.

[17] F. W. Grover, Inductance Calculations: Working Formulas and Tables (Dover Publications, New York, NY, 1946).

[18] O. A. Hurricane, J. Appl. Phys. 95, 4503 (2004).

[19] J. M. Creedon, J. Appl. Phys. 48, 1070 (1977).

[20] Alexander L. Velikovich, Igor V. Sokolov, and Andrey A. Esaulov, Phys. Plasmas 9, 1366 (2002).

[21] M. L. Kiefer, K. L. Fugelso, K. W. Struve, and M. M. Widner, User's Guide for Version 2.0 (Sandia National Laboratory, Albuquerque, NM, 1995).

[22] Mike Lopez, Y. Y. Lau, John W. Luginsland, David W. Jordan, and Ronald M. Gilgenbach, Phys. Plasmas 10, 4489 (2003).

[23] A. A. Kim, M. G. Mazarakis, V. A. Sinebryukhov, B. M. Kovalchuk, V.A. Visir, S. N. Volkov, F. Bayol, A. N. Bastrikov, V. G. Durakov, S. V. Frolov, V. M. Alexeenko, D. H. McDaniel, W. E. Fowler, K. LeChien, C. Olson, W. A. Stygar, K.W. Struve, J. Porter, and R.M. Gilgenbach, Phys. Rev. ST Accel. Beams 12, 050402 (2009).

[24] J. C. Martin on Pulsed Power, edited by T. H. Martin, A. H. Guenther, and M. Kristiansen (Plenum Press, New York, 1996), p. 267.

[25] Simon C. Bott, David M. Haas, Yossof Eshaq, Utako Ueda, Robert E. Madden, Gilbert Collins IV, and Farhat N. Beg, IEEE Trans. Plasma Sci. 38, 567 (2010).

[26] R. E. Madden, S. C. Bott, G. Collins IV, and F. N. Beg, IEEE Trans. Plasma Sci. 37, 433 (2009). 